\title{
Effects of Treating an Overactive Urinary Bladder in Patients with Multiple Sclerosis
}

\author{
Maida Zonić-Imamović ${ }^{1}$, Semir Imamović ${ }^{2}$, Amela Čičkušić ${ }^{1}$, Azra Delalić ${ }^{1}$ \\ Renata Hodžić3 ${ }^{3}$ Mirza Imamović ${ }^{4}$
}

${ }^{1}$ Clinic for Physical Medicine and Rehabilitation, University Clinical Center Tuzla, Bosnia and Herzegovina, ${ }^{2}$ Clinic of Anesthesiology and Resuscitation, University Clinical Center Tuzla, Bosnia and Herzegovina, ${ }^{3}$ Clinic of Neurology, University Clinical Center Tuzla, Bosnia and Herzegovina, ${ }^{4}$ Medical Faculty, Univerity of Tuzla, Bosnia and Herzegovina

Correspondence: maidazo@yahoo.com

Tel.: + 38761728728

Fax.: + 38735303148

Received: 6 October 2019; Accepted: 27 December 2019

\begin{abstract}
Objective. The purpose of this study was to evaluate the efficiency of the anticholinergic therapy with oxybutynin and the effects of daily transcutaneous tibial nerve stimulation (TTNS) on the quality of life of patients with an overactive bladder (OAB) and multiple sclerosis (MS). Patients and Methods. The study was designed as a randomized controlled trial. The patients who suffer from MS underwent urodynamic tests which showed that they had an OAB. The tests used to assess symptoms and quality of life were Overactive Bladder Questionnaires (OAB-q) SF. Patients were divided into 2 groups of 30 patients each. The first group received a $5 \mathrm{mg}$ oxybutynin tablet twice a day for 3 months and the second group had TTNS every day for 3 months. Results. The anticholinergic therapy showed a statistically significant improvement in all symptoms and quality of life $(\mathrm{P}<0.001)$. Side effects such as dry mouth were observed in about $35 \%$ of patients. The results of the study TTNS daily therapy showed good performance in the reduction all clin-
\end{abstract}

ical symptoms of the bladder and improved quality of life, with statistical significance $(\mathrm{P}<0.05)$ and with no side effects. It was found that the improved quality of life parameters and the reduced symptoms were more statistically significant in the treatment with oxybutynin tablets than TTNS therapy $(\mathrm{P}<0.001)$. Conclusion. Our recommendation for the treatment of $\mathrm{OAB}$ is oxybutynin in doses of $2 \times 5 \mathrm{mg}$. If a patient can not tolerate anticholinergic drugs, daily TTNS is recommended to reduce OAB symptoms and improve quality of life, without side effects.

Key Words: Overactive Bladder - Oxybutynin TTNS.

\section{Introduction}

Difficulties in urination/micturition are frequent in patients with multiple sclerosis (MS), cause health problems and decrease the patients' quality of life (1). Up to $80 \%$ of newly diagnosed patients, as well as up to $96 \%$ of those who have suffered from MS for more than 10 years, have problems with urination (2). MS is a disease which mostly affects people between 20 and 40 years old at the time of diagnosis. It rarely affects people before the age of 15 and after 60 (3). Women are affected twice as often as men, but men have more severe symptoms. The most common voiding disturbance in patients with multiple sclerosis is a spastic bladder or overactive bladder (OAB) (4). An overactive bladder is defined as the occurrence of 
bladder contractions suddenly, without the person having control over it. Overactive bladder syndrome is sometimes called an irritable bladder or detrusor instability (5).

The progression of neurological diseases results in the deterioration of overactive bladder symptoms. A correct diagnosis and treatment can improve the control of the bladder and the patients' quality of life (5). The International Continence Society (ICS) defines the overactive bladder syndrome as an urgency to urinate, with or without urge incontinence and an increased frequency of urination during the day and night (6). Medicines in the class of drugs called antimuscarinics (also called anticholinergics) may alleviate the symptoms of an overactive bladder. They act by blocking certain nerve impulses to the detrusor, which relaxes the detrusor thereby increasing bladder capacity. Different types of anticholinergics, with similar effects on the symptoms of an overactive bladder, are used. The side-effects of these are dependent on the dose given. The medication with certain side effects mentioned in the research was oxybutynin $(7,8)$. Oxybutynin chloride is a moderate anticholinergic and severe musculotropic drugs. It is one of the first drugs which has been used for more than 30 years in the treatment of $\mathrm{OAB}$ patients and, until recently, the only one available in our country. In our study we investigated the effects of oxybutynin chloride (Ditropan) in the $2 \times 5 \mathrm{mg}$ doses, which is a half of the maximum daily dose. The most common side-effects are dry mouth, dry eyes, constipation, blurred vision and drowsiness.

In addition to anticholinergics, transcutaneous tibial nerve stimulation (TTNS) is also used in the treatment of $\mathrm{OAB}$, using the TENS apparatus. TENS-Transcutaneous electrical nerve stimulation is a non-invasive exponential current (low-frequency current) method that efficiently suppresses the excessive activity of the urinary bladder. Nervus tibialis is a mixed nerve composed of motor and sensory nerve fibers that originate from roots L4 to S3 and stimulate the innervation of the bladder, urinary sphincter and pelvic floor by stimulating the nociceptors of spinothalamic tract neurons and reducing or eliminating excessive activity (9).

The purpose of this study was to evaluate the efficiency of the anticholinergic therapy with $2 \times 5 \mathrm{mg}$ doses of oxybutynin, and then the effects of daily transcutaneous tibial nerve stimulation (TTNS) on the quality of life of patients with an overactive bladder $(\mathrm{OAB})$ and multiple sclerosis.

\section{Patients and Methods}

The study was a randomized controlled trial, that tested the efficacy of oxybutynin anticholinergic therapy and daily transcutaneous tibial nerve stimulation (TTNS) on the quality of life of patients with an overactive bladder (OAB) and multiple sclerosis (MS). Participants were enrolled consecutively as they were visiting the Urodynamics unit of the Physical Medicine and Rehabilitation Clinic, University Medical Center Tuzla for a period of 2 years.

The inclusion criteria were patients with MS who uderwent urodynamic testing that showed an $\mathrm{OAB}$ with maximum bladder capacity of less than $300 \mathrm{ml}$. Each patient should have had a normal urine finding before the urodynamic testing. Exclusion criteria were symptoms lasting for less than 6 months, pregnancy, sacral peripheral nerve lesions, urinary tract infections, serious secondary illnesses and detrusor-sphincter dyssynergy-determined by a urodinamic investigation. Patients were divided into 2 groups of 30 patients each. The first group received a $5 \mathrm{mg}$ oxybutynin tablet twice a day for 3 months and the second group had TTNS every day for 3 months.

Medio tens (Iskra Medical) devices were administered to stimulate the nervous tibials posterior and were administered to patients at 
home daily. Current intensity, frequency, and pulse duration were set on each apparatus at a frequency of $10 \mathrm{HZ}$, constant stimulation with intervals of 200 microseconds and duration of stimulation of $30 \mathrm{~min}$. The active selfadhesive electrode (A) was placed behind the internal malleolus and the other $10 \mathrm{~cm}$ above the right ankle. Patients were given detailed instructions, how the sensation should be, how to operate the device at home with the above parameters already set. All patients who received daily stimulation were given a device to use at home for a period of 3 months.

$\mathrm{OAB}-\mathrm{q} \mathrm{SF}$ is a specific questionnaire used to assess the frequency of hyperactive bladder symptoms and the quality of life of patients with urinary disorders and consists of 2 subscales. The symptom subscale contains 6 questions rated individually from 1 to 6 and quality of life subscale includes 13 questions rated from 1 to 6 .

The symptoms which were monitored and affected the quality of life are: urgency (>than 3 to $24 \mathrm{~h}$ ), daily voiding (frequency $>8$ in $24 \mathrm{~h}$ ), nocturia and urgent incontinence. The OAB-q SF questionnaire for determining symptoms and quality of life was completed before therapy and 3 months after therapy.

\section{Statistical Analysis}

Statistical analyses were performed using MedCalc software (ver.12.1.4.0 for Windows; MedCalc, Mariakerke, Belgium). To test the differences between the groups in terms of quantitative variables, the paired $t$ test and Wilcoxon sum rank tests were done, depending on data distribution. $\mathrm{P}<0.05$ was considered significant.

\section{Results}

Sixty patients were examined. There were $33.3 \%$ men and $66.6 \%$ women. The subjects receiving the anticholinergic drug oxybutynin were on average $45.8 \pm 8.13$ years old with a disease duration of $8.3 \pm 5.1$ years, and the subjects who had electrostimulation n. tibialis with TENS were on average $47.36 \pm 7.98$ years old with a disease duration of $8.9 \pm 4.9$ years. $(\mathrm{P}>0.05)$.

The stimulation with TENS therapy has shown a statistically significant reduction in all clinical symptoms of the bladder and an improved quality of life. The symptom score before treatment with daily TTNS was $61.2 \pm 14.6$ and decreased after treatment to $50.8 \pm 12.3(\mathrm{P}=0.004)$. The quality of life score increased from $28.5 \pm 12.6$ after daily TTNS treatment to $38.3 \pm 11.4 \quad(\mathrm{P}=0.003)$ (Figura 1). The anticholinergic therapy with oxybutynin produced a statistically significant improvement in all symptoms and quality of life.

The symptom score before the oxybutynin treatment was $61.9 \pm 6$ and $32.4 \pm 14.8$ $(\mathrm{P}<0.001)$ after treatment. The quality of life score before the oxybutynin treatment was $27.8 \pm 13.7$ and $56.1 \pm 17.3(\mathrm{P}<0.001)$ after treatment.

The therapy with oxybutinin tablets and stimulation with TENS therapy have shown a statistically significant reduction in all clinical symptoms of the bladder and an improvement in quality of life. After comparing the two types of treatment, it was concluded that there were no statistically significant improvements in the number of daily mycions in favor of the treatment with oxybutynin tablets $(\mathrm{P}=0.021)$, and no statistically significant difference in the number of nocturnal, daytime and nocturnal incontinence groups $(\mathrm{P}>0.05)$ (Table 1$)$.

After comparing the quality of life and symptoms measured by the OAB-q SF questionnaire by treatment type, it was found that the improved quality of life parameters and the reduced symptoms were more statistically significant in the treatment with oxybutynin tablets than TTNS therapy $(\mathrm{P}<0.001)$. The side effect of a dry mouth after oxybutynin therapy was found in $35 \%$ of patients, which did not require discontinu- 


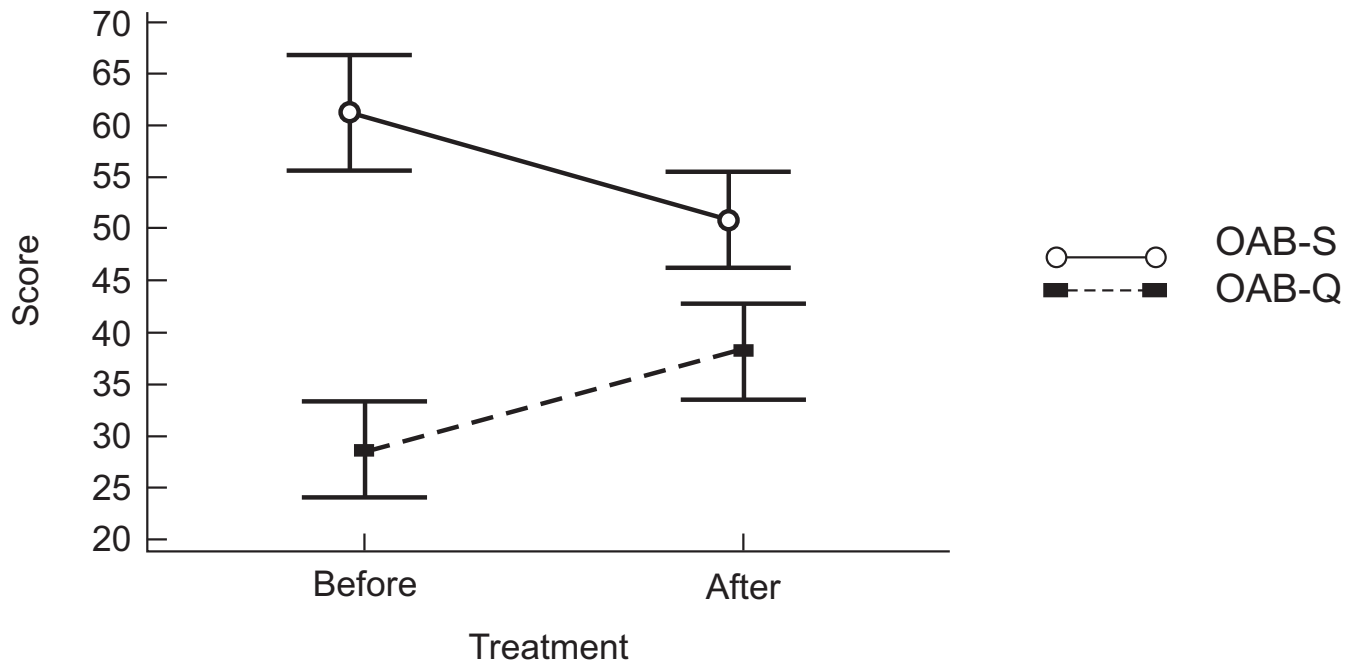

Figure 1. Symptom score and quality of life before and after the treatment with daily daily transcutaneous tibial nerve stimulation $(\mathrm{P}<0.05)$; $\mathrm{OAB}-\mathrm{S}=$ Overactive bladder symptoms, $\mathrm{OAB}-\mathrm{Q}=$ Overactive bladder quality of life.

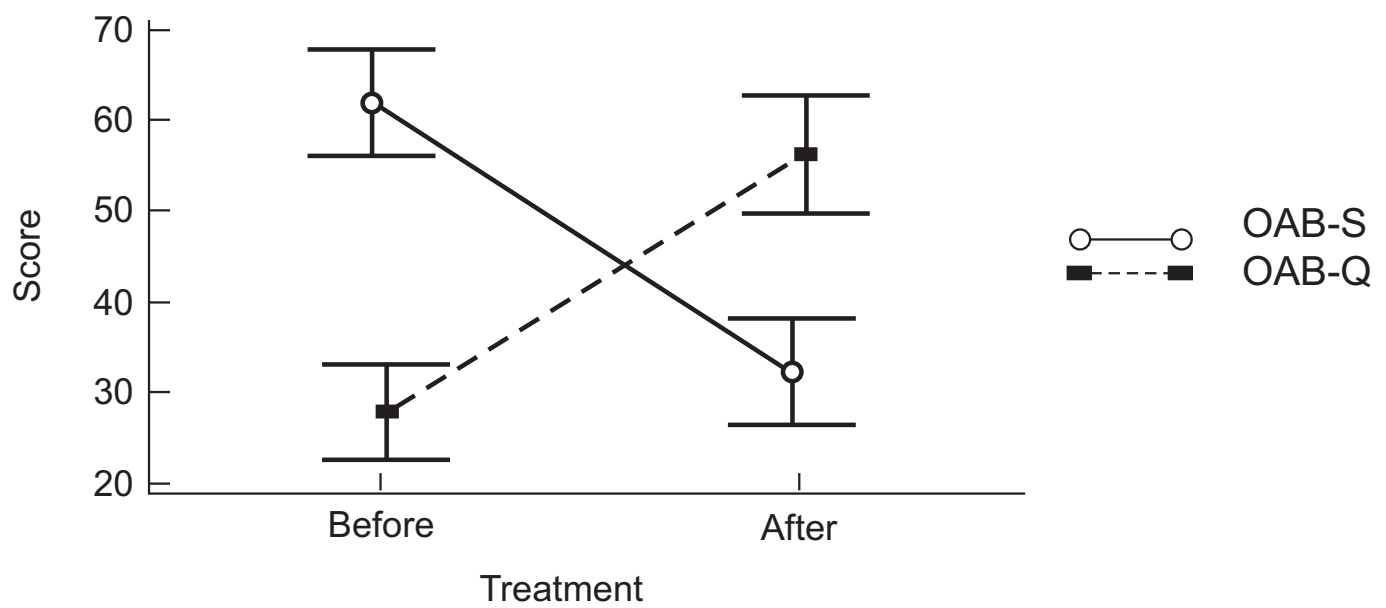

Figure 2. The symptom scores and quality of life before and after treatment with Oxybutynin $(\mathrm{P}<0.0001)$; $\mathrm{OAB}-$ $\mathrm{S}=$ Overactive bladder symptoms, $\mathrm{OAB}-\mathrm{Q}=$ Overactive bladder quality of life.

Table 1. The Comparison of Clinical Symptoms after the Oxybutynin Treatment and Daily Tibial Nerve Stimulation

\begin{tabular}{llll}
\hline \multirow{2}{*}{ Clinical symptoms } & \multicolumn{2}{l}{ Treatment } & $\mathrm{P}^{\dagger}$ \\
\cline { 2 - 4 } & $\begin{array}{l}\text { Oxybutynin } \\
(\bar{x} \pm \mathrm{SD})\end{array}$ & $\begin{array}{l}\text { TTNS } \\
(\bar{x} \pm \mathrm{SD})\end{array}$ & 0.021 \\
\hline Daily micturition number & $7.5 \pm 2.8$ & $9.4 \pm 3.4$ & 0.729 \\
\hline Night micturition number & $2.2 \pm 1.6$ & $2.3 \pm 1.4$ & 0.112 \\
\hline Daily incontinence number & $1.7 \pm 1.7$ & $2.4 \pm 1.4$ & 0.719 \\
\hline Night incontinence number & $0.6 \pm 0.8$ & $0.6 \pm 0.5$ & $<0.001$ \\
\hline OAB-Q & $56.1 \pm 17.2$ & $38.3 \pm 11.4$ & $<0.001$ \\
\hline OAB-S & $32.4 \pm 14.8$ & $50.8 \pm 12.3$ & \\
\hline
\end{tabular}

*Transcutaneous tibial nerve stimulation; +Paired t-test; $\mathrm{OAB}-\mathrm{S}=$ Overactive bladder symptoms, $\mathrm{OAB}-\mathrm{Q}=\mathrm{Overactive} \mathrm{bladder}$ quality of life. 
ation of therapy. There were no side effects after the TTNS therapy.

\section{Discussion}

In our study, oxybutnin at a dose of $10 \mathrm{mg}$ daily, divided into two doses, was shown to be a very effective drug in the treatment of an overactive bladder, where the parameters of the quality of life and the symptoms of overractive bladder were significantly improved. Alhasso et al., (10) searched papers related to the treatment of an overactive bladder with anticholinergics in MS using the Cochrane database. Thirteen surveys were reviewed with 1770 participants and all surveys were designed as parallel groups except one that was a cross-over study. The duration of treatment was 3 to 12 weeks, with one check 24 weeks after starting treatment. It was concluded that the use of anticholinergic drugs in the treatment of $\mathrm{OAB}$ produces good results. The results are better when using anticholinergics compared to bladder training, as well as the two modalities compared with each modality individually. There is insufficient data to show the effects of therapy after discontinuation of anticholinergics. Anticholinergics are known to have side effects, such as dry mouth. Side effects such as dry mouth have been reported in about $1 / 3$ of patients using anticholinergic therapy. In our study, we found similar results with regard to the side effects where $35 \%$ of patients had a dry mouth but problems were not expressed and therapy was not interrupted.

Although the dose used in this study was half the maximum, it still achieved a significant improvement in all parameters measured by the urodynamic study. Similar results were shown by Rolf et al. (11) who have shown that flexible dosing of anticholinergics in the treatment of $\mathrm{OAB}$ is a useful strategy in clinical practice to achieve maximum effects with a maximum level of tolerance. A study was conducted with 1658 patients who used $15 \mathrm{mg}$ trospium chloride (TID) or $2.5 \mathrm{mg}$ oxybutynin 3 times a day, gradually increasing the dose. By the end of the study the dose was $90 \mathrm{mg}$ TID and $15 \mathrm{mg}$ oxybutynin daily. A decrease in incontinence episodes and frequency of urination was observed. Dry mouth was less pronounced in the TID group than the Oxybutynin group at $15 \mathrm{mg}$ daily. The conclusion was that the incidence of incontinence and number of mictions could be significantly reduced by a flexible dose of the drug to prevent side effects such as dry mouth. In this study, TID was shown to be better tolerated than oxybutynin at a dose of $15 \mathrm{mg}$. Similar results were demonstrated by Yoo et al. (12) who tested the appropriate dose of oxybutinin in clinical practice. The study included 809 patients, of whom 590 received treatment for 12 weeks. They used a different dose of oxybutynin from $5 \mathrm{mg}$ to $30 \mathrm{mg}$. Most patients received 5 or $10 \mathrm{mg}$ oxybutinin daily. Symptom assessment was performed with POSQ and all symptoms improved. Similarly, drug dosing was done in a study by MacDiarmid et al. (13). The conclusion was that the dose should be flexible with the need to constantly monitor the effectiveness of the treatment and side effects.

Versi et al. (14) examined the occurrence of the side effect of dry mouth with the conventional administration of oxybutnin and slow release oxybutninin. They gradually increased the dose from $5 \mathrm{mg}$ in both groups to a total of $20 \mathrm{mg}$ daily. The incidence of dry mouth increased with increasing dose of oxybutinin in both groups. Thomas et al. (15) investigated the effect of various antimuscarinic (anticholinergic) drugs in $\mathrm{OAB}$ treatment by meta-analysis. A study of 69 studies concluded that antimuscarinic drugs used at the recommended doses, presented individually for each drug, had a similar effect, and it was emphasized that oxybutnin at a dose greater than $10 \mathrm{mg}$ per day had more pronounced side effects than other drugs. A 
similar study was conducted by Hay-Smith et al., (16) where they reviewed 49 studies in a meta-analysis to find out which anticholinergic drug should be used to treat $\mathrm{OAB}$ in adults. It was concluded that it is better to use lower doses as much as possible, which have an effect, to avoid side effects, which was also proven in our study. There was little or no available evidence of the quality of life, cost, or the long-term outcome in these studies.

As antihilonergic drugs have been used for the treatment of $\mathrm{OAB}$ for many years, it has become necessary to find new treatments that will improve bladder function with as few side effects as possible. Given the choice of a slightly invasive method (PTNS) or non-invasive one (TTNS), most studies are focused on using this treatment method (17). In our study, we monitored the effect of TTNS therapy in patients who had daily stimulation.

The results of the study with daily stimulation showed good efficacy in reducing all clinical symptoms of the bladder, especially the number of nocturnal incontinence, all of which had a positive effect on the quality of life. TTNS therapy also showed good tolerability. The effectiveness of the therapy was observed as early as the second week of treatment and did not change significantly over the 3 months of treatment duration.

The efficacy as well as tolerance of the therapy provides a great opportunity for patients, especially if they have other additional diseases or symptoms that would prevent them from using anticholinergics. This therapy gives them the ability to be treated without fear of side effects. Voorham et al., (18) demonstrated the acute effect of a single TTNS application in patients with $\mathrm{OAB}$ symptoms using urodynamic parameters. Out of the 40 patients, 20 only underwent the urodynamics test, and TTNS stimulation was performed on the other group of 20 patients during the urodynamics test with 1 electrode per $n$. tibialis and the other on the S2-S4 foramen for $20 \mathrm{~min}$. The results of the urodynamic parameters for the first group without stimulation were almost unchanged, and in the group after stimulation there was a statistically significant improvement $(\mathrm{P}<0.05)$ in bladder capacity, volume of mycation and the first contraction of the detrusor. The study was performed as a diagnostic procedure rather than a treatment.

In France, a multicenter study was conducted in which 70 patients treated with the TTNS technique suffering from MS were monitored (19). Stimulation with TTNS lasted 3 months and the duration of therapy was 20 minutes per day. They used the Qualiveen questionnaire to assess the quality of life. After treatment, $83.3 \%$ of patients experienced a statistically significant improvement in clinical parameters and symptoms, as well as the quality of life.

\section{Conclusion}

Adequate diagnosis and treatment can improve bladder control for patients with symptoms of $\mathrm{OAB}$. Our recommendation for the treatment of OAB is an anticholinergic (Oxybutynin) in doses of $2 \times 5 \mathrm{mg}$ as it produces good results for all parameters (symptoms) and quality of life. It is unfortunate that electrostimulation therapy which is non-invasive and has no side effects is used so rarely, and produces good results. If patients for any reason can not tolerate anticholinergic drugs, daily TTNS is recommended to reduce OAB symptoms and improve quality of life, without side effects.

\section{What Is Already Known on this Topic:}

There are a number of ways to treat $O A B$, and pharmacological treatment remains the most important in the management of patients with $O A B$ symptoms. Anticholinergics reduce contractility and increase bladder capacity, and the difficulty in prescribing medications for $O A B$ is due to a lack of drug selectivity leading to unwanted side effects. In addition to pharmacological therapy in the treatment of $O A B$, electrical tibial nerve stimulation is used, which is a non-invasive exponential current (low-frequency) method that suppresses excessive bladder activity. 
What this Study Adds:

In this study, we confirmed the effect of pharmacological therapy in the treatment of $O A B$, but also the positive effects of under-utilized electrostimulation, which are non-invasive and have no side effects. The disadvantage of this study is the shortterm follow-up of patients, and there are no studies on the $d u$ ration of therapy.

Authors' Contributions: Conception and design: MZI, SI and MI; Acquisition, analysis and interpretation of data: MZI, AD, RH and AČ; Drafting the article: MZI, AČ and MI; Revising it critically for important intellectual content: MZI, AD, AČ and SI; Approved final version of the manuscript: MZI, SI, AČ, $\mathrm{AD}, \mathrm{RH}$ and $\mathrm{MI}$.

Conflict of Interest: The authors declare that they have no conflict of interest.

\section{Reference}

1. Cianco SJ, Mutchnik SE, Rivera VM, Boone TB. Urodynamic pattern changes in multiplesclerosis. Urology. 2001;57(2):239-45.

2. Foster H. Bladder symptoms and multiple sclerosis. Mult Scler Quart Rep. 2002;21(1):1-34.

3. Wingerchuk DM, Lucchinetti CF, Noseworthy JH. Multiple sclerosis: current pathophysiological concept. Lab Invest. 2001;81(3):263-81.

4. Akkoç Y, Ersöz M, Yüceyar N, Tunç H, Köklü K, Yoldaş TK, et al. Overactive bladder symptoms in patients with multiple sclerosis: Frequency, severity, diagnosis and treatment. Neurogenic Bladder Turkish Research Group. J Spinal Cord Med. 2016;39(2):229-33.

5. Kalsi V, Fowler C. Therapy Insight: Bladder Dysfunction Associated With Multiple Sclerosis. Nat Clin Pract Urol. 2.2005;(10):492-501.

6. Abrams P, Artibani W, Cardozo L, Dmochowski R, van Kerrebroeck P, Sand P. Terminology Report: The Ongoing Debate. Neurourol Urodyn. 2006;25:293

7. Zinner N, Tuttle J, Marks L. Efficacy and tolerability of darifenacin, a muscarinic M3 selective receptor antagonist (M3 SRA), compared with oxybutynin in the treatment of patients with overactive bladder. World J Urol. 2005;23(4):248-52.

8. Chapple CR, Abrams P. Comparison of darifenacin and oxybutynin in patients with overactive bladder: assessment of ambulatory urodynamics and impact on salivary flow. Eur Urol. 2005;48(1):102-9.

9. Vandoninck V, van Balken MR, Finazzi Agrò E, Petta F, Micali F, Heesakkers JP, et al. Percutane- ous tibial nerve stimulation in the treatment of overactive bladder: urodynamic data. Neurourol Urodyn. 2003;22(3):227-32.

10. Alhasso AA, McKinlay J, Patrick K, Stewart L. Anticholinergic drugs versus non-drug active therapies for overactive bladder syndrome in adults. Cochrane Database of Systematic Reviews, 2006;Issue 4. Art. No.: CD003193.

11. Rolf-Hasso B, Helmut M, Claudia N, Michael Z. Dose escalation improves therapeutic outcome: post hoc analysis of data from a 12-week, multicentre, double-blind, parallel-group trial of trospium chloride in patients with urinary urge incontinence. BMC Urol. 2010;10:15.

12. Yoo D, Han J, Lee K, Choo M. Prescription pattern of oxybutynin ER in patients with overactive bladder in real life practice: a multicentre, open-label, prospective observational study. Int J Clin Pract. 2012;66(2):132-8.

13. MacDiarmid SA, Anderson RU, Armstrong RB, Dmochowski RR. Efficacy and safety of extended release oxybutynin for the treatment of urge incontinence: an analysis of data from 3 flexible dosing studies. J Urol. 2005;174(4 Pt 1):1301-5.

14. Versi E, Appell R, Mobley D, Patton W, Saltzstein D. Dry mouth with conventional and controlledrelease oxybutynin in urinary incontinence. The Ditropan XL Study Group. Obstet Gynecol. 2000;95(5):718-21.

15. Kessler TM, Bachmann LM, Minder C, Löhrer D, Umbehr M, Schünemann HJ, et al. Adverse event assessment of antimuscarinics for treating overactive bladder: a network meta-analytic approach. LoS POne. 2011;23;6(2):e16718.

16. Hay-Smith J, Herbison P, Ellis G, Morris A. Which anticholinergic drug for overactive bladder symptoms in adults. Cochrane Database Syst Rev. 2005;20;(3):CD005429. Review. Update in: Cochrane Database Syst Rev. 2012;1:CD005429.

17. Kozma B, Majoros A, Pytel Á, Póka R, Takács P. Efficacy of the percutaneous tibial nerve stimulation in the treatment of lower urinary tract symptoms. Orv Hetil. 2018;159(43):1735-40.

18. Voorham-van der Zalm P, Elzevier H, Guus AB, Nijeholt L, Pelger R. Simultaneous Sacral and Tibial Transcutaneous Electrical Nerve Stimulation: urodynamic evaluation Current Urology. 2007;1(2):77-80.

19. De Seze M, Raibaut P, Gallien P, Even-Schneider A, Denys P, Bonniaud V, et al. Transcutaneous posterior tibial nerve stimulation for treatment of the overactive bladder syndrome in multiple sclerosis: results of a multicenter prospective study. Neurourol Urodyn. 2011;30(3):306-11. 